\title{
Highly expressed IMP3 and GLUT-1 in combination with the loss of BAP1 expression is useful for differentiating malignant mesothelioma from reactive mesothelial hyperplasia
}

\section{Hongjun Zhu}

Affiliated Hospital of Nantong University

\section{Qing Hu}

the third affiliated hospital of nantong university

Daishan Jiang

Affiliated Hospital of Nantong University

Hua Huang

Affiliated Hospital of Nantong University

Jian Liu

Affiliated Hospital of Nantong University

Jianguo Zhang

Affiliated Hospital of Nantong University

\section{Tingting Bian}

Affiliated Hospital of Nantong University

Yifei Liu ( $\square$ ntdxliuyifei@sina.com)

Affiliated Hospital of Nantong University

\section{Research}

Keywords: BAP1, IMP3, GLUT-1, immunohistochemistry, malignant mesothelioma, reactive mesothelial hyperplasia

Posted Date: February 20th, 2020

DOI: https://doi.org/10.21203/rs.2.24122/v1

License: (1) This work is licensed under a Creative Commons Attribution 4.0 International License. Read Full License 


\section{Abstract}

Background Our study aims to evaluate how IHC staining of BRCA1 associated protein-1 (BAP1), insulinlike growth factor mRNA binding protein 3 (IMP3), and glucose transporter-1 (GLUT-1) can help to differentiate $M M$ from reactive mesothelial hyperplasia $(\mathrm{RMH})$.

Methods The expression levels of BAP1, IMP3, and GLUT-1 were investigated using immunohistochemistry (IHC) in $38 \mathrm{MMs}$ and $32 \mathrm{RMHs}$.

Results BAP1 was lost in 20 (52.6\%) of the 38 MMs. IMP3 and GLUT-1 were positive in $26(68.4 \%)$ and 25 (65.8\%), respectively, of the $38 \mathrm{MMs}$. The results of IHC analyses of BAP1, IMP3, and GLUT-1 indicated that these markers were characterized by a $100 \%$ specificity with sensitivities of $52.63,68.42$, and $65.79 \%$, respectively. In addition, BAP1, IMP3, and GLUT-1 combined showed $100 \%$ specificity and the highest sensitivity (81.58\%). Meanwhile, loss of BAP1 expression is related to a better prognosis.

Conclusions Overexpressed IMP3 and GLUT-1 in combination with the loss of BAP1 showed high specificity to MM that was different from that in $\mathrm{RMH}$ and improved diagnostic accuracy, especially in MM.

\section{Background}

Malignant mesothelioma (MM) is the most common mesothelioma with a poor prognosis and a median survival of 9.2-11.2 months [1-3]. Unlike $M M$, reactive mesothelial hyperplasia $(R M H)$ is a self-limiting disease often caused by injury to the mesothelial surface; therefore, it is important to differentiate the two conditions to be able to accurately treat the patient and provide a prognosis. Although $\mathrm{RMH}$ is a benign condition, in some cases, especially in small biopsy tissues and effusion, it is difficult to identify benign from malignant mesothelial cells [4]. Current guidelines on pathology and cytology recommend the use of immunohistochemistry $(\mathrm{IHC})$ and molecular detection for a differential diagnosis $[5,6]$. Although IHC's usefulness has been fully demonstrated, the specificity and sensitivity of $\mathrm{IHC}$ markers are different. Some IHC markers, such as IMP3 [7-9] and GLUT-1 [9-12] have become the most popular of antibodies because of their relatively high diagnostic performance. In recent years, the loss of BAP1 expression in mesothelial cells has also become a marker with high specificity of malignant tumors.

BAP1 encodes BRCA1-related protein 1 (BAP1), which is a nuclear localization deubiquitinase that regulates gene expression, transcription, and DNA repair and plays a role in tumor inhibition by enhancing the inhibition of BAP1-mediated cell proliferation [13]. The deletion or inactivation of BAP1 may be caused by a chromosome deletion in BAP1 locus at 3p21.1 or by the sequence variation in BAP1, which has been proved to be associated with various tumors, including MM [14].

IMP3 is a carcinoembryonic, cancer-specific protein involved in embryogenesis, and its expression is associated with many malignant tumors related to aggressive and advanced cancers; it is not expressed 
in benign tissues [15-17]. IMP3 has also been shown to promote the proliferation, invasion, and metastasis of tumor cells and may play an important role in the diagnosis of MM [18-20].

GLUT-1 is a glucose transporter with a high affinity and low capacity and is found mainly in the plasma membrane and usually expressed in red blood cells [21, 22], peripheral nerves [23], renal tubules [24], the blood-brain barrier [25], and placental tissues [26]. Although not expressed in most normal epithelial cells or epithelial benign tumors, GLUT-1 is expressed in epithelial malignancies in various organs [27-29]. Using IHC to detect GLUT-1 has shown great potential in differentiating MM from RMH [11].

The purpose of this study was to evaluate how IHC staining (i.e., immunostaining) of BAP1, IMP3, and GLUT-1 cells can help to differentiate MM from RMH.

\section{Materials And Methods Case selection}

Tissue samples from 38 patients with MM and 32 with RMH who were treated from January 2000 to December 2016 at the Affiliated Hospital of Nantong University, China, were collected from the hospital's pathology department. All of the selected cases had identical diagnostic opinions and a definite diagnosis by two pathologists. Patients having an inconsistent diagnosis were excluded from the study. The Human Research Ethics Committee of the hospital was informed of the content of this investigation and approved the study. All samples used were anonymous archival specimens, and informed consent was not required for this study.

\section{IHC procedures and evaluation of BAP1, IMP3, and GLUT-1 expression}

IHC staining was conducted using the following antibodies: anti-BAP1 mouse monoclonal antibody (clone C-4; Santa Cruz Biotechnology, Santa Cruz, CA, USA; 1:100 dilution), anti-IMP3 rabbit monoclonal antibody (clone EP286; Maixin, Fuzhou, China; 1:100 dilution), and anti-GLUT-1 mouse monoclonal antibody (clone SPM498; Thermo Fisher Scientific, Kalamazoo, Ml; 1:200 dilution). Nonmesothelial cells that were immunoreactive to BAP1 (e.g., inflammatory cells such as histiocytes and lymphocytes, fibroblasts, pneumocytes, and endothelial cells) served as internal positive controls for each staining protocol. Analysis of BAP1 after IHC revealed staining in the nucleus, and BAP1 loss in the tumor cells was defined as nuclear staining at an intensity lower than that of the internal positive control [30].

We set the cutoff value at $50 \%$ for IHC analysis of BAP1 as previously described [31]. IMP3 cytoplasmic/ membranous staining and GLUT- 1 membranous staining in $\geq 10 \%$ of the target cells were considered positive. Lymphoid cells for IMP3 and red blood cells for GLUT-1 served as the positive internal controls [32].

\section{Statistical analyses}


Fisher's exact test was used to evaluate the staining differences in BAP1, IMP3, and GLUT-1 among the $\mathrm{MM}$ and $\mathrm{RMH}$ cells. The corresponding receiver operating characteristic (ROC) curves were plotted for different combinations of immunostains, and the areas under these correlated ROC curves (AUCs) were compared using the nonparametric approach of DeLong et al [33]. The survival curve was calculated using the Kaplan-Meier method. $P<0.05$ was considered statistically significant. Statistical analyses were performed using SPSS ver. 24 (IBM Corp., Armonk, NY, USA).

\section{Results}

\section{Clinicopathological characteristics}

Among the 38 patients with MM, 30 were males (M:F = 15:4). The average age was 61.87 years (range, 35-82 years). Thirty-eight MM specimens comprised 20 that were surgically excised, 12 that were biopsied, and 6 that were identified as pleural effusion cell masses. There were 32 cases of RMH, 24 of which were benign pulmonary mesothelial reactive hyperplasia, and 8 that were among the pleural effusion cell mass specimens (28 males and 4 females; average age 56.16 years; range, 21-76 years).

\section{IHC results}

The results of IHC staining of BAP1, IMP3, and GLUT-1 are summarized in Table 1. BAP1 was lost in 20 (52.6\%) of the 38 MMs. IMP3 and GLUT-1 were positive in $26(68.4 \%)$ and $25(65.8 \%)$, respectively, of the 38 MMs. There was no IMP3 or GLUT-1 expression in any of the 32 cases of RMH. 
Table 1

Diagnostic application of detection assays to differentiate malignant mesothelioma from reactive mesothelial hyperplasia.

\begin{tabular}{|c|c|c|c|}
\hline & & $M M(n=38)$ & $\mathrm{RMH}(\mathrm{n}=32)$ \\
\hline \multirow[t]{2}{*}{ BAP1 IHC } & $\mathrm{R}$ & 18 & 32 \\
\hline & $\mathrm{L}$ & 20 & 0 \\
\hline \multirow[t]{2}{*}{ GLUT1 IHC } & $P$ & 25 & 0 \\
\hline & $\mathrm{N}$ & 13 & 32 \\
\hline \multirow[t]{2}{*}{ IMP3 IHC } & $P$ & 26 & 0 \\
\hline & $\mathrm{N}$ & 12 & 32 \\
\hline \multirow[t]{2}{*}{ BAP1/GLUT1 } & $\mathrm{L} / \mathrm{P}$ & 28 & 0 \\
\hline & $\mathrm{R} / \mathrm{N}$ & 10 & 32 \\
\hline \multirow[t]{2}{*}{ BAP1/ IMP3 } & $\mathrm{L} / \mathrm{P}$ & 28 & 0 \\
\hline & $\mathrm{R} / \mathrm{N}$ & 10 & 32 \\
\hline \multirow[t]{2}{*}{ BAP1/GLUT1/ IMP3 } & $L / P / P$ & 31 & 0 \\
\hline & $\mathrm{R} / \mathrm{N} / \mathrm{N}$ & 7 & 32 \\
\hline \multicolumn{4}{|c|}{ MM: malignant mesothelioma; RMH: reactive mesothelial hyperplasia; R: Retained; L:Loss; } \\
\hline \multicolumn{4}{|c|}{ P: Positive; N: Negative. } \\
\hline
\end{tabular}

\section{Sensitivity and specificity of detection assays for discriminating MM from RMH}

Representative hematoxylin and eosin (H\&E) stains of BAP1, IMP3, and GLUT-1 IHC assays of the MM and RMH samples are shown in Figs. 1 and 2. The sensitivity and specificity of each detection assay for discriminating MM from RMH samples are summarized in Table 2. All IHC assays of BAP1, IMP3, and GLUT-1 showed $100 \%$ specificity with sensitivities of $52.63,68.42$, and $65.79 \%$, respectively. IHC assays for all combinations of BAP1, IMP3, and GLUT-1 also showed 100\% specificity. The sensitivity of BAP1 alone was low with IHC, but increased when combined with other markers, and the combination of BAP1, IMP3, and GLUT-1 gave the highest sensitivity at $81.58 \%$ (Fig. 3A). 
Table 2

Sensitivity and Specificity of IHC in malignant mesothelioma and reactive mesothelial hyperplasia.

\begin{tabular}{|llll|}
\hline & Sensitivity $(95 \% \mathrm{Cl})$ & Specificity $(95 \% \mathrm{Cl})$ & AUC (95\% Cl) \\
\hline BAP1 & $52.63(35.8-69.0)$ & $100(89.1-100.0)$ & $0.763(0.646-0.857)$ \\
\hline GLUT1 & $65.79(48.6-80.4)$ & $100(89.1-100.4)$ & $0.829(0.720-0.908)$ \\
\hline IMP3 & $68.42(51.3-82.5)$ & $100(89.1-100.5)$ & $0.842(0.735-0.918)$ \\
\hline B\&G\&I & $81.58(65.7-92.3)$ & $100(89.1-100.3)$ & $0.908(0.815-0.964)$ \\
\hline B\&G\&I: BAP1\& GLUT1 \&IMP3. & & \\
\hline
\end{tabular}

\section{The ROC curves for the IHC markers}

AUCs (95\% confidence interval [CI]) for BAP1, IMP3, and GLUT-1 were $0.763(0.646-0.857), 0.842$ (0.735$0.918)$, and $0.829(0.720-0.908)$, respectively. AUC ( $95 \% \mathrm{Cl})$ for BAP1, IMP3, and GLUT-1 combined was 0.908 (0.815-0.964) (Table 2, Fig. 3B).

\section{Survival analyses}

Kaplan-Meier analysis showed that loss of BAP1 expression is associated with a better prognosis in MM $(P=0.0217$; Fig. 4A). The prognosis was not statistically different for IMP3, and GLUT-1 in MM $(P=0.187$, $P=0.332$; Fig. 4B,C).

\section{Discussion}

This study demonstrated the IHC assays and analyses of BAP1, IMP3, and GLUT-1 that can be used to differentiate MM from RMH. BAP1 is a newly identified diagnostic marker whose loss is specific to MM $[34,35]$. Previous studies have found BAP1 loss in $27-67 \%$ of MM $[30,31,36]$, more commonly in epithelioid MM (68-77\%) than in sarcomatoid/desmoplastic MM (0-22\%) [37-39]. The results of our study showed that BAP1 was lost in $20(52.6 \%)$ of the $38 \mathrm{MMs}$, which is similar to the previous findings in that the loss of BAP1 expression is usually not seen in RMH proliferation [37]. Previous studies have observed, together with ours, $100 \%$ specificity for BAP1 after the IHC assay in MM with a sensitivity of $52.63 \%$. In addition, AUC for BAP1 was 0.763 (0.646-0.857). Meanwhile, BAP1 mutation or loss of BAP1 expression has been shown to be associated with a better prognosis in MM [3]. Of course, our study came up with the same results.

IMP3 was first identified in a screening for pancreatic carcinoma-specific markers [40] and was noted to be highly expressed in both MMs and lung carcinomas [8, 41]. Positive levels of IMP3 were reported to have a wide range from 53 to $93 \%$. Our study showed that IMP3 positivity was $68.4 \%$ in MM. The sensitivity and specificity of IMP3 from IHC staining results distinguishing between MMs and benign 
mesothelial proliferations were reported as ranging from 36 to $91 \%$ and 73 to $100 \%$, respectively. We observed that the sensitivity and specificity of IMP3 were 68.42 and $100 \%$, respectively; therefore, our results are consistent with those of previous reports. AUC of IMP3 (0.842) was higher than that for other individual markers.

GLUT-1 is a high-affinity glucose transporter that is expressed in normal human tissues, including red blood cells, the endothelia of the blood-brain barrier, and the placenta [42, 43]. GLUT-1 also appears to be upregulated in certain types of malignancies, including those of lung [44], breast [45], head and neck (squamous) [46], and ovary [47]. In our study, we found that GLUT-1 was expressed in 25 of the 38 cases of MM. Lagana et al.[48] have found that 73/135 (54\%) of malignant tumors were positive for GLUT-1. In our study and those of Kato et al. [11] and Lagana et al.,[48] GLUT-1 did not test positive in the RMH specimens. Previous studies have reported that GLUT-1 sensitivity ranged from 40 to $100 \%$, while its specificity ranged from 63 to $100 \%$ in MMs $[8,9,11,32]$. In our study, GLUT-1 sensitivity and specificity were 65.79 and $100 \%$, respectively, and AUC of GLUT-1 was 0.829 .

\section{Conclusions}

In the present study, we examined the results of IHC assays of BAP1, IMP3, and GLUT-1 and related these to their levels in $\mathrm{MM}$ and $\mathrm{RMH}$. We hypothesized that evaluating all markers together could improve sensitivity. Our study showed that using a BAP1, IMP3, and GLUT-1 panel improved sensitivity by up to $81.58 \%$, and that the sensitivity of the combined BAP1, IMP3, and GLUT-1 was significantly higher than that in any other combination of stains. Meanwhile, we also found that AUC of the combined BAP1, IMP3, and GLUT-1 was the highest at $0.908(P<0.05)$.

Overexpressing IMP3 and GLUT-1 in combination with BAP1 loss was highly specific in differentiating MM from RMH. Combining BAP1, IMP3, and GLUT-1 improved diagnostic accuracy, especially that in MM.

\section{Study Limitations}

There were some limitations to the present study. First, the study comprised a relatively small number of cases. Second, the expression of these markers in different types of MM was not analyzed. Third, although several studies have demonstrated that BAP1 loss is associated with better survival and is an independent prognostic factor, we did not study the correlation between the expression of the markers and the prognosis of patients with MM. The shortcomings of our study are the result of the small number of cohorts; a study with a larger cohort is needed to draw more definitive conclusions.

\section{Abbreviations}

MM: Malignant mesothelioma; BAP1: BRCA1 associated protein-1; IMP3: insulin-like growth factor mRNA binding protein 3; GLUT-1: glucose transporter-1; RMH: reactive mesothelial hyperplasia 


\section{Declarations}

\section{Acknowledgements}

Not applicable.

\section{Funding}

This study was funded by grants from Key Scientific and Technological Projects in Nantong of Jiangsu (No.MS22018001, MS22019015), Jiangsu Post-doctoral Foundation Research Project (No.2019Z142), Nantong Science and technology plan project (No.MSZ19164) and Nantong university clinical medicine special clinical basic research youth project (2019JQ001).

\section{Authors' contributions}

$\mathrm{HZ}$, QH designed the study and drafted the manuscript. DJ participated in data analysis. HH performed the evaluation of the IHC stains. JZ and JL provided tissue specimens. BT and YL financed the study and participated in evaluation of the IHC stains. All authors reviewed the manuscript for important intellectual content. All authors read and approved the final manuscript.

\section{Availability of data and materials}

Is available upon request from the corresponding author.

\section{Ethics approval and consent to participate}

Ethics approval and consent to participate was given by all patients in writing (ethics approval was given by the Ethics Committee of Affiliated Hospital of Nantong University).

\section{Competing interests}

The authors declare that they have no competing interests.

\section{Author details}


${ }^{1}$ Department of Pathology, Affiliated Hospital of Nantong University, Nantong 226001, China.

${ }^{2}$ Department of Oncology, The third affiliated hospital of Nantong university, Nantong 226001, China.

${ }^{3}$ Department of Thoracic Surgery, Affiliated Dongtai Hospital of Nantong University, Dongtai, 224200, Jiangsu, China.

${ }^{4}$ Department of Emergency Medicine, Affiliated Hospital of Nantong University, Nantong 226001, China. ${ }^{5}$ Department of Chemotherapy, Affiliated Hospital of Nantong University, Nantong 226001, China

\section{References}

1. Beckett P, Edwards J, Fennell D, Hubbard R, Woolhouse I, Peake MD. Demographics, management and survival of patients with malignant pleural mesothelioma in the National Lung Cancer Audit in England and Wales. Lung Cancer. 2015;88(3):344-8.

2. Damhuis RA, Khakwani A, Schutter HD, Rich AL, Burgers JA, Meerbeeck JPV. Treatment patterns and survival analysis in 9014 patients with malignant pleural mesothelioma from Belgium, the Netherlands and England. Lung Cancer. 2015;89(2):212-7.

3. Farzin M, Toon CW, Clarkson A, Sioson L, Watson N, Andrici J, et al. Loss of expression of BAP1 predicts longer survival in mesothelioma. Pathology. 2015;47(4):302-7.

4. Churg A, ., Colby TV, , Cagle P, ., et al. The separation of benign and malignant mesothelial proliferations. Am J Surg Pathol. 2000;24(9):1183-200.

5. Husain AN, Colby TV, Ordó?Ez NG, Allen TC, Attanoos RL, Beasley MB, et al. Guidelines for Pathologic Diagnosis of Malignant Mesothelioma: 2017 Update of the Consensus Statement From the International Mesothelioma Interest Group. Arch Pathol Lab Med. .

6. Hjerpe A, Ascoli V, Bedrossian C, Boon M, Creaney J, Davidson B, et al. Guidelines for cytopathologic diagnosis of epithelioid and mixed type malignant mesothelioma. Complementary statement from the International Mesothelioma Interest Group, also endorsed by the International Academy of Cytology and the Papanicolaou Society of. Diagn Cytopathol. 2015;43(7):563-76.

7. King J, ., Thatcher N, ., Pickering $C$, ., et al. Sensitivity and specificity of immunohistochemical antibodies used to distinguish between benign and malignant pleural disease: a systematic review of published reports. Histopathology. 2010;49(6):561-8.

8. Lee AF, , Gown AM, , Andrew C. IMP3 and GLUT-1 immunohistochemistry for distinguishing benign from malignant mesothelial proliferations. Am J Surg Pathol. 2013;37(3):421-6.

9. Hiroshi M, Nozomu K, Mana F, Takayuki N, Katsuo U, Motoyasu S, et al. Comparative immunohistochemical analysis of IMP3, GLUT1, EMA, CD146, and desmin for distinguishing malignant mesothelioma from reactive mesothelial cells. Am J Clin Pathol. 2014;141(1):85-93.

10. Husain AN, , M Kamran M, Allen G, Kenzo H, Yiqing C, et al. How useful is GLUT-1 in differentiating mesothelial hyperplasia and fibrosing pleuritis from epithelioid and sarcomatoid mesotheliomas? An international collaborative study. Lung Cancer. 2014;83(3):324-8. 
11. Kato Y, Tsuta K, Seki K, Maeshima AM, Watanabe S, Suzuki K, et al. Immunohistochemical detection of GLUT-1 can discriminate between reactive mesothelium and malignant mesothelioma. Mod Pathol. 2007;20(2):215-20.

12. Monaco SE, , Yongli S, Mona B, Krasinskas AM, , et al. The diagnostic utility of p16 FISH and GLUT-1 immunohistochemical analysis in mesothelial proliferations. Am J Clin Pathol. 2011;135(4):619-27.

13. Ventii $\mathrm{KH}$, , Devi NS, , Friedrich KL, , et al. BRCA1-associated protein-1 is a tumor suppressor that requires deubiquitinating activity and nuclear localization. Cancer Res. 2008;68(17):6953.

14. Carbone M, Yang H, Pass HI, Krausz T, Testa JR, Gaudino G. BAP1 and cancer. Nat Rev Cancer. 2013;13(3):153.

15. Hoffmann NE, , Yuri S, Lohse CM, , Parker AS, et al. External validation of IMP3 expression as an independent prognostic marker for metastatic progression and death for patients with clear cell renal cell carcinoma. Cancer. 2008;179(4):133-4.

16. Zhong J, Chu PG, , Woda BA, , Qin L, et al. Combination of quantitative IMP3 and tumor stage: a new system to predict metastasis for patients with localized renal cell carcinomas. Clinical Cancer Research An Official Journal of the American Association for Cancer Research. 2008;14(17):5579.

17. Jiang Z, Lohse CM, Chu PG, Wu CL, Woda BA, Rock KL, et al. Oncofetal protein IMP3: a novel molecular marker that predicts metastasis of papillary and chromophobe renal cell carcinomas. Cancer. 2008;112(12):2676.

18. Baisong L, Yan H, Herrick DJ, , Gary B. The RNA-binding protein IMP-3 is a translational activator of insulin-like growth factor II leader-3 mRNA during proliferation of human K562 leukemia cells. J Biol Chem. 2005;280(18):18517-24.

19. Jonas V, Hansen TVO, , Lars J, Rehannah B, Wewer UM, et al. RNA-binding IMPs promote cell adhesion and invadopodia formation. EMBO J. 2014;25(7):1456-68.

20. Findeis-Hosey JJ, , Haodong X. The use of insulin like-growth factor II messenger RNA binding protein-3 in diagnostic pathology. Hum Pathol. 2011;42(3):303-14.

21. Wann JG, Lin CS, Chang LC, Hsu YH, Chien CT, Tai DW, et al. Enhanced Expression of Glucose Transporter 1 on Erythrocyte Membrane in Hemodialysis Patients: The Possible Role in Erythrocyte Ascorbate Recycling. Am J Kidney Dis. 2006;47(6):1055-63.

22. GarcãA-Alvarez I, Garrido L, Fernã nA. Studies on the uptake of glucose derivatives by red blood cells. ChemMedChem. 2010;2(4):496-504.

23. Salla JT, , Johann ACBR, , Lana AMA, , et al. Immunohistochemical study of GLUT-1 in oral peripheral nerve sheath tumors. Oral Dis. 2010;14(6):510-3.

24. Linden KC, Dehaan CL, Zhang Y, Glowacka S, Cox AJ, Kelly DJ, et al. Renal expression and localization of the facilitative glucose transporters GLUT1 and GLUT12 in animal models of hypertension and diabetic nephropathy. Am J Physiol Renal Physiol. 2006;290(1):F205.

25. Kavi D, Klinger ME, , Myers RL, , Ashwini M, et al. GLUT-1 glucose transporters in the blood-brain barrier: differential phosphorylation. J Neurosci Res. 2011;89(12):1913-25. 
26. Baumann MU, Deborde S, Illsley NP. Placental glucose transfer and fetal growth. Endocrine. 2002;19(1):13-22.

27. Patiño-Seijas B, Lorenzo-Franco F, Rey-Sanjurjo JL, González-Cuesta M. Vascular Lesions: GLUT-1 Expression as a Diagnostic Tool to Discriminate Tumors From Malformations. J Oral Maxillofac Surg. 2012;70(10):2333-42.

28. Sasaki H, Shitara M, Yokota K, Hikosaka Y, Moriyama S, Yano M, et al. Overexpression of GLUT1 correlates with Kras mutations in lung carcinomas. Mol Med Rep. 2012;5(3):599.

29. Kobayashi M, Kaida H, Kawahara A, Hattori S, Kurata S, Hayakawa M, et al. The relationship between GLUT-1 and vascular endothelial growth factor expression and 18F-FDG uptake in esophageal squamous cell cancer patients. Clin Nucl Med. 2012;37(5):447-52.

30. Kinoshita Y, Hida T, Hamasaki M, Matsumoto S, Sato A, Tsujimura T, et al. A combination of MTAP and BAP1 immunohistochemistry in pleural effusion cytology for the diagnosis of mesothelioma. Cancer. 2017;126.

31. Yoshimura M, Kinoshita Y, Hamasaki M, Matsumoto S, Hida T, Oda Y, et al. Diagnostic application of BAP1 immunohistochemistry to differentiate pleural mesothelioma from metastatic pleural tumors. Histopathology. 2017;71(6).

32. Sunhee C, Mee-Hye O, Sun-Young J, Joungho H, Tae-Jung K, Minseob E, et al. Practical utility of insulin-like growth factor II mRNA-binding protein 3, glucose transporter 1, and epithelial membrane antigen for distinguishing malignant mesotheliomas from benign mesothelial proliferations. Pathol Int. 2015;64(12):607-12.

33. Delong ER, , Delong DM, , Clarke-Pearson DL, . Comparing the areas under two or more correlated receiver operating characteristic curves: a nonparametric approach. Biometrics. 1988;44(3):837-45.

34. Testa JR, , Mitchell C, Jianming P, Below JE, , et al. Germline BAP1 mutations predispose to malignant mesothelioma. Nat Genet. 2011;43(10):1022.

35. Matthew B, Marie B, Taylor BS, , Shigeki S, Tatsuo I, et al. The nuclear deubiquitinase BAP1 is commonly inactivated by somatic mutations and 3p21.1 losses in malignant pleural mesothelioma. Nat Genet. 2011;43(7):668-72.

36. Pillappa R, Maleszewski JJ, Sukov WR, Bedroske PP, Greipp PT, Boland JM, et al. Loss of BAP1 Expression in Atypical Mesothelial Proliferations Helps to Predict Malignant Mesothelioma. Am J Surg Pathol. 2017;42(2):256.

37. Cigognetti M, Lonardi S, Fisogni S, Balzarini P, Pellegrini V, Tironi A, et al. BAP1 (BRCA1-associated protein 1 ) is a highly specific marker for differentiating mesothelioma from reactive mesothelial proliferations. Mod Pathol. 2015;28(8):1043-57.

38. Hwang HC, Pyott S, Rodriguez S, Cindric A, Carr A, Michelsen C, et al. BAP1 Immunohistochemistry and p16 FISH in the Diagnosis of Sarcomatous and Desmoplastic Mesotheliomas. Am J Surg Pathol. 2016;40(5):714.

39. Righi L, Duregon E, Vatrano S, Izzo S, Giorcelli J, Rondón-Lagos M, et al. BRCA1-Associated Protein 1 (BAP1) Immunohistochemical Expression as a Diagnostic Tool in Malignant Pleural Mesothelioma 
Classification: A Large Retrospective Study. J Thorac Oncol. 2016;11(11):2006-17.

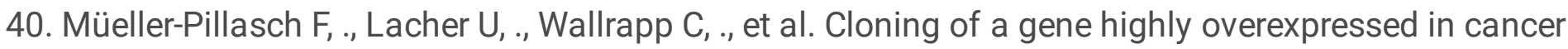
coding for a novel KH-domain containing protein. Oncogene. 1997;14(22):2729-33.

41. Yan J, Wei Q, Jian W, Qiu B, Wen J, Liu J, et al. IMP3 Predicts Invasion and Prognosis in Human Lung Adenocarcinoma. Lung. 2015;194(1):137-46.

42. Farrell CL, , Yang J, ., Pardridge WM, . GLUT-1 glucose transporter is present within apical and basolateral membranes of brain epithelial interfaces and in microvascular endothelia with and without tight junctions. J Histochem Cytochem. 1992;40(2):193-9.

43. Thorens B. Glucose transporters in the regulation of intestinal, renal, and liver glucose fluxes. Am J Physiol Gastrointest Liver Physiol. 1996;270(4 Pt 1):G541.

44. Ito $T$, Noguchi $Y$, Satoh $S$, Hayashi $H$, Inayama $Y$, Kitamura $H$. Expression of facilitative glucose transporter isoforms in lung carcinomas: its relation to histologic type, differentiation grade, and tumor stage. Mod Pathol. 1998;11(5):437-43.

45. Brown RS, , Wahl RL, . Overexpression of Glut-1 glucose transporter in human breast cancer. An immunohistochemical study. Cancer. 2015;72(10):2979-85.

46. Reisser C, ., Eichhorn K, ., Herold-Mende C, ., et al. Expression of facilitative glucose transport proteins during development of squamous cell carcinomas of the head and neck. Int $\mathrm{J}$ Cancer. 2015;80(2):194-8.

47. Cantuaria G, ., Fagotti A, ., Ferrandina G, ., et al. GLUT-1 expression in ovarian carcinoma: association with survival and response to chemotherapy. Cancer. 2001;92(5):1144-50.

48. Lagana SM, Taub RN, Borczuk AC. Utility of glucose transporter 1 in the distinction of benign and malignant thoracic and abdominal mesothelial lesions. Arch Pathol Lab Med. 2012;136(7):804-9.

\section{Figures}



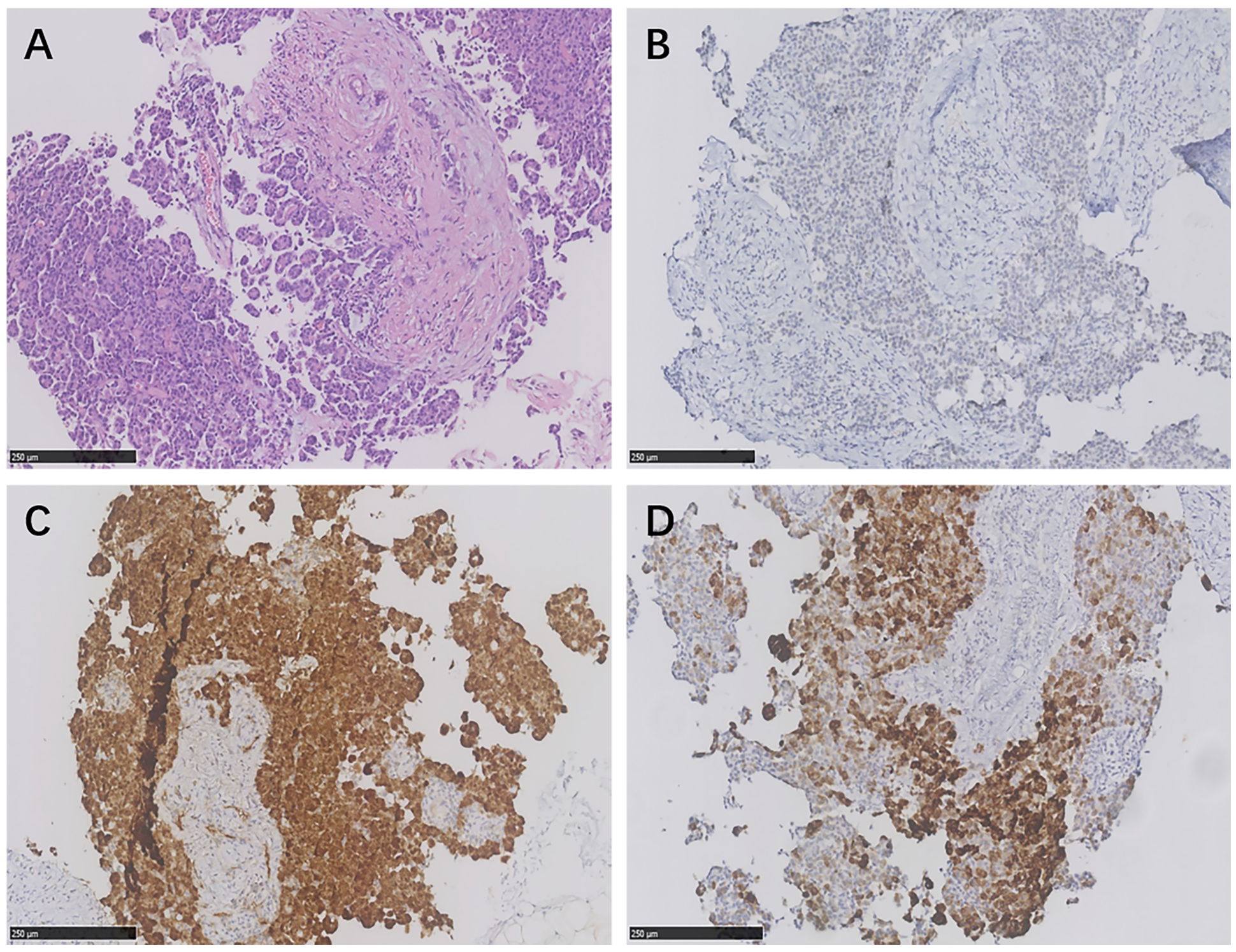

\section{Figure 1}

Representative examples of H\&E staining (A) and BAP1 (B), IMP3 (C), and GLUT-1(D) immunostaining in MM. Original magnifications $\times 200$ (A-D). (A): epithelioid MM, (B): loss BAP1 expression, (C): high IMP3 expression (cytoplasmic/ membranous staining), (D) high GLUT-1 expression (membranous staining). 

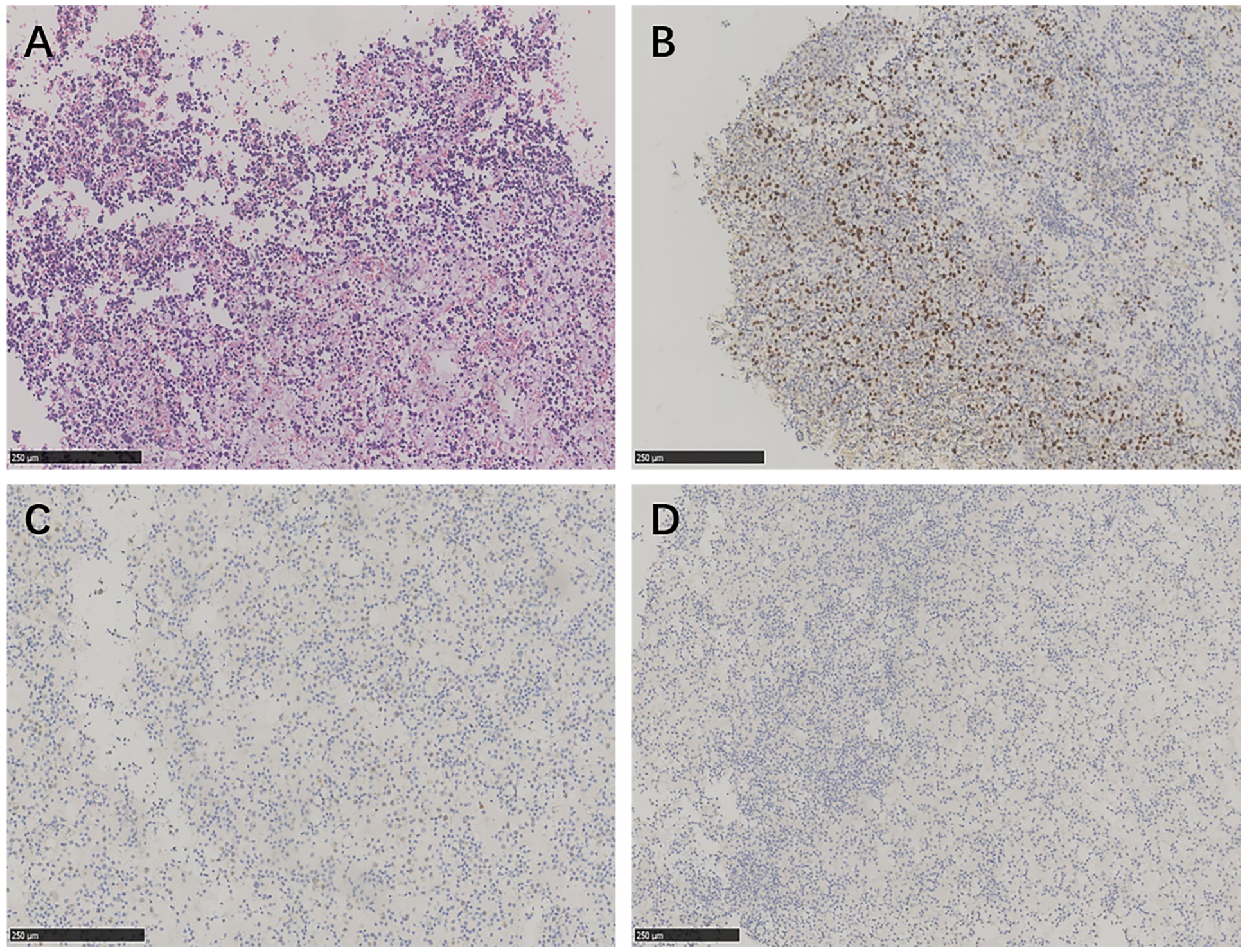

\section{Figure 2}

Representative examples of H\&E staining (A) and BAP1 (B), IMP3 (C), and GLUT-1(D) immunostaining in RMH. Original magnifications $\times 200(A-D)$. (A): Reactive mesothelial hyperplasia, (B): retained BAP1 expression, (C): negative IMP3 expression, (D) negative GLUT-1 expression. 

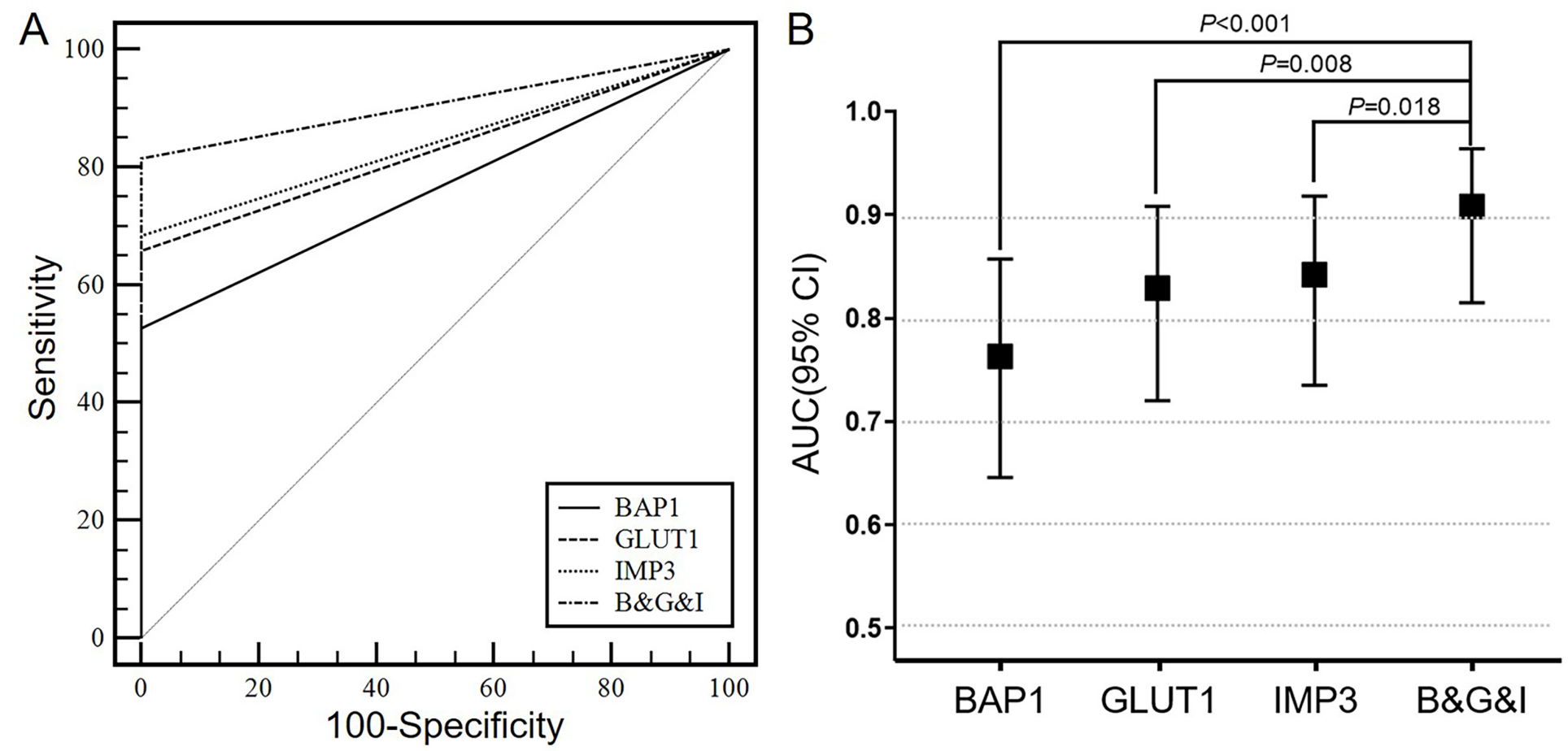

\section{Figure 3}

The ROC curves for the IHC markers. (A): Sensitivity and specificity of BAP1, GLUT1, IMP3, BAP1\& GLUT1 \&IMP3 for discriminating MM from RMH, (B): AUC (95\% Cl) of BAP1, GLUT1, IMP3, BAP1\& GLUT1 \&IMP3 for discriminating $\mathrm{MM}$ from $\mathrm{RMH}$.
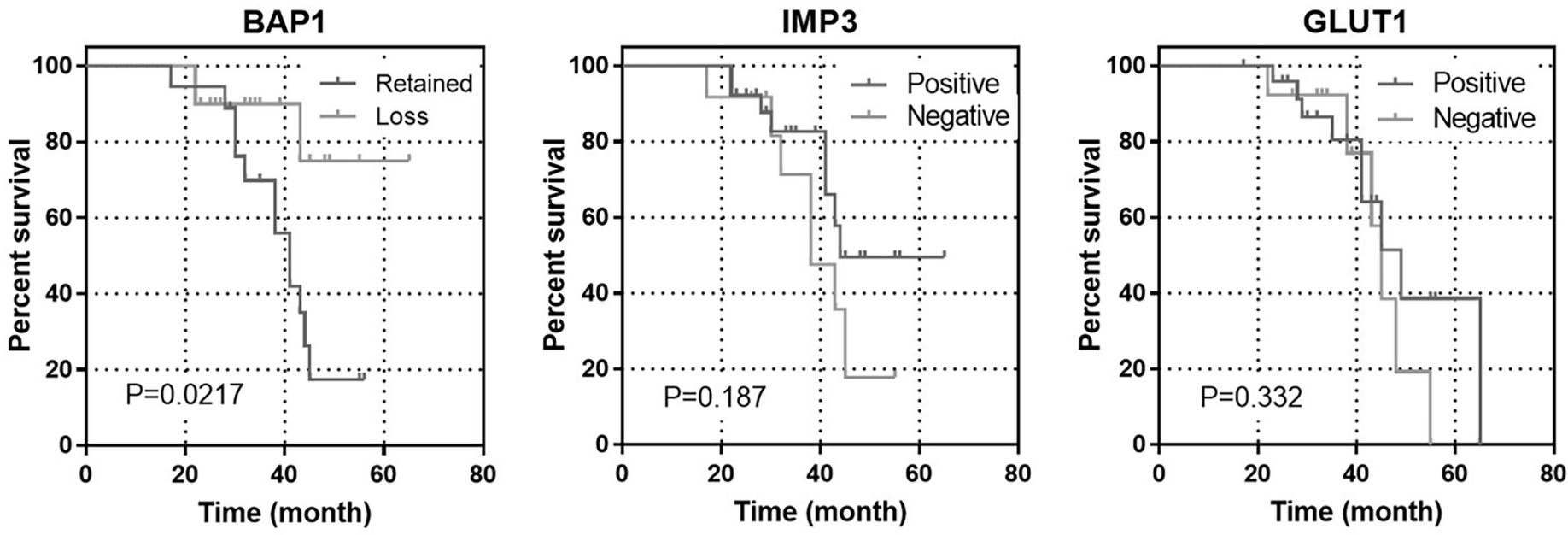

Figure 4

Overall survival (OS) curves for patients with MM. (A): BAP1 status, (B): IMP3 status, (C): GLUT-1 status. 